\title{
DEPTHS OF CUT IDENTIFICATION IN 3-AXIS MILLING USING CUTTING FORCE SPECTRUM
}

\author{
L. Morelli ${ }^{1 *}$, N. Grossi ${ }^{1}$, A. Scippa ${ }^{1}$, G. Campatelli ${ }^{1}$ \\ ${ }^{1}$ University of Firenze, Department of Industrial Engineering, Via di Santa Marta 3, 50139, Firenze, Italy \\ ${ }^{*}$ Corresponding author; e-mail: lorenzo.morelli@unifi.it
}

\begin{abstract}
Cutting forces analysis is one of the most important means of improving machining quality and productivity. In 3-axis milling, the frequency domain analysis of the cutting forces provides information which can be adopted for both predicting and monitoring the cutting process. However, the evaluation of the cutting forces spectra requires the application of specific tools (i.e., Fourier's Transform) to the time domain representation of the same cutting forces. This paper presents analytical formulations to directly evaluate the cutting force spectra starting from the tool geometry and the cutting parameters. The proposed formulations start from the expression of the cutting forces as a Fourier series and enhance the predictions showed in previous works by reducing the overall number of calculations needed. Then, the proposed formulations are applied to identify both radial and axial depths of cut exploiting the normalized cutting force spectrum. Finally, the proposed formulations and their application were numerically and experimentally validated. This work represents a preliminary step in the development of a monitoring system to identify cutting conditions in 3-axis milling.
\end{abstract}

\section{Keywords:}

End milling; Simulation; Frequency; Cutting Force; Monitoring.

\section{INTRODUCTION}

In machining, the analysis of cutting forces is essential to increase both quality and productivity of the process [Liu 2002]. Indeed, the prediction of cutting forces is crucial to handle both static phenomena (i.e., static deflection [Nishida 2018; Grossi 2019]) and dynamic issues (i.e., vibrations [Budak 2003; Grossi 2017]).

For 3-axis milling, many studies have been proposed concerning the prediction of cutting forces [Kapoor 1997; Matsumura 2017; Kiran 2019; Ducroux 2021]. In this context, mechanistic approaches have been largely used for their ease of implementation [Rubeo 2016]. The mechanistic modeling assumes that instantaneous cutting forces depend on the uncut chip area and empirical coefficients (i.e., cutting coefficients) which are function of tool edge geometry, and tool/workpiece material properties. In literature, the mechanistic models may adopt one single cutting coefficient (i.e., lumped shear force model), which includes the effects of both shearing and ploughing [Altintas 1995] or two separate cutting coefficients (i.e., dualmechanism force model), one to include the effect of shearing (i.e., cutting coefficient) and the other (i.e., edge coefficient) to include the effect of ploughing [Budak 1996]. With the lumped shear force model, cutting force prediction is simplified, but the accuracy of the prediction is limited. On the other hand, in the dual-mechanism force model, the use of two empirical coefficients allows a more accurate force prediction despite the higher complexity.

In general, mechanistic models are used in the time domain [Campatelli 2012], and they have been exploited to identify radial and axial depth of cut with the aim of controlling and monitoring the cutting process. Indeed, the knowledge of depths of cuts during the cutting process allows to detect and avoid unwanted cutting conditions. For example, Altintas et al. [Altintas 1987] used mechanistic cutting force model to identify the radial width and the axial depth of cut from two orthogonal force measurements in the plane perpendicular to the machine tool spindle. The method of the authors does not require any knowledge about cutting coefficients, but it requires the evaluation of several constants with calibration cuts. Instead, Jong-Geun Choia et al. [Choi 1999] proposed an algorithm to estimate the axial depth of cut from the cutting force pattern. Thank to this the magnitude of cutting force could be neglected; however, the approach does not fully investigate the cutting force pattern limiting its reliability. On the other hand, Yang et al. [Yang 2004] defined analytical force indices to link depth of cut variations with force shape characteristics in end-milling. The author's approach is effective, but it requires cutting coefficients and several calculations. Moreover, nominal depths of cut must be known to obtains their deviations. Instead, Leal-Munoz et al. [Leal-Muñoz 2018] showed a technique to identify axial and radial depth of cut in finishing operations using entry and exit times of the cutting edge. Despite the high accuracy of the identified depths of cut, the approach is limited to cutting operations with high axial depth of cut and only one cutting edge involved.

All the mentioned approaches are based on time domain cutting forces, while very few methods rely on cutting forces in frequency domain. Indeed, Wang et al [Junz Wang 2002] used the convolution analysis to present a closed form for the cutting force spectrum in both feed and cross-feed 
direction. Later, the same authors [Wang 2003] used these formulations to identify tool run-out. On the other hand, Bachrathy et al [Bachrathy 2016] used the used the analytical Fourier series expression for the cutting forces in the frequency domain to evaluate the tool motion caused by forced vibrations.

Despite the analytical approach, no direct formulations for the Fourier coefficients were provided. Instead, Schmitz et al. [Schmitz 2006] expressed as Fourier series the cutting forces in the cross-feed direction and provided analytical formulations for each coefficient of the series compute surface location error. However, these formulations require several calculations to consider the contribute of both the tool helix and the tool flutes.

This paper follows the approach presented by Schmitz et al. and provides enhanced formulations to express the Fourier coefficients that describes the cutting force spectrum in both feed and cross-feed directions. Then, these formulations are applied to the identification of the radial and the axial depth of cut starting from the cutting forces in the frequency domain. The proposed idea has been numerically and experimentally validated, and it represents a starting point in the development of a cutting condition monitoring approach.

\section{CUTTING FORCE FREQUENCY CONTENT}

The following method aims at predicting the frequency content of cutting forces using analytical formulations, without requiring the simulation of cutting forces in timedomain. In this work the formulations are proposed for 3 axis milling considering a dual mechanism force model, that relates forces linearly to chip thickness with a cutting coefficient and includes the ploughing effect via edge coefficients. Focusing on tangential and radial forces, the resulting cutting forces in time-domain are:

$$
\begin{aligned}
& F_{t}(\phi)=\sum_{j=1}^{N} K_{t c} a_{p} h\left(\phi_{j}\right)+K_{t e} a_{p} \\
& F_{r}(\phi)=\sum_{j=1}^{N} K_{r c} a_{p} h\left(\phi_{j}\right)+K_{r e} a_{p}
\end{aligned}
$$

where $K_{t c}, K_{r c}$ are the tangential and radial cutting coefficients, $K_{t e}, K_{r e}$ are the tangential and radial edge coefficients, $N$ is the number of flutes, $a_{p}$ is the axial depth of cut and $h$ is the chip thickness that can be computed as:

$h=f_{z} \sin \left(\phi_{j}\right)$

where:

$$
\begin{aligned}
& \phi=w t \\
& \phi_{j}=\phi+\left(\frac{2 \pi}{N}\right)(j-1) j=1,2, \ldots, N
\end{aligned}
$$

$\mathrm{f}_{\mathrm{z}}$ is the feed per tooth, $t$ is the time variable, and $w$ is the spindle speed in rad/s. Cutting force in feed ( $x$ ) and crossfeed direction $(\mathrm{y})$ can be derived as follows:

$$
\begin{aligned}
& F_{x}(\phi)=-F_{t} \cos \left(\phi_{j}\right)-F_{r} \sin \left(\phi_{j}\right) \\
& F_{y}(\phi)=F_{t} \sin \left(\phi_{j}\right)-F_{r} \cos \left(\phi_{j}\right)
\end{aligned}
$$

It must be pointed out that these formulations in timedomain should be evaluated only in the range between the cutter entry angle $\left(\phi_{\text {in }}\right)$ and the cutter exit angle $\left(\phi_{\text {out }}\right)$ which are identified according to both cutting strategy and the radial depth of cut $\left(a_{r}\right)$ with these expressions:

$$
\begin{aligned}
\phi_{\text {in }} & =\pi-\alpha_{\text {en }} \text { Down-milling; } & \phi_{\text {in }} & =0 \text { Up-milling } \\
\phi_{\text {out }} & =\pi \text { Down-milling; } & \phi_{\text {out }} & =\alpha_{\text {en }} \text { Up-milling }
\end{aligned}
$$

where $D$ is the tool diameter and:

$\alpha_{e n}=\operatorname{acos}\left(2 a_{r} / D\right)$

The proposed approach starts from the formulation in frequency domain proposed by Schmitz et al. [Schmitz 2006] for $F_{y}$.

$$
F_{y}(\phi)=\sum_{j=1}^{N}\left(a_{y 0}+\sum_{n=1}^{\infty}\left(a_{y n} \cos n \phi_{j}+b_{y n} \sin n \phi_{j}\right)\right)
$$

where $a_{0} \ldots a_{n}$ are the Fourier series coefficients that, for the purpose of this work, can be written as:

$$
a_{y 0}=a_{y 0}^{*} \cdot a_{p} ; a_{y n}=a_{y n}^{*} \cdot a_{p} ; \quad b_{y n}=b_{y n}^{*} \cdot a_{p}
$$

where $a^{*} y_{0}$ represents the Fourier coefficients at zero frequency, while $a_{y n}^{*}, b_{y n}^{*}$ are two coefficients which define the real and imaginary components of the force at the frequency n-multiple of the rotation frequency, divided for the axial depth of cut. The same formulations can be obtained for $F_{X}$ with different coefficients.

$$
\begin{aligned}
& F_{x}(\phi)=\sum_{j=1}^{N}\left(a_{x 0}^{*} \cdot a_{p}+\sum_{n=1}^{\infty}\left(a_{x n}^{*} \cdot a_{p} \cos n \phi_{j}+b_{x n}^{*} .\right.\right. \\
& \left.\left.a_{p} \sin n \phi_{j}\right)\right)
\end{aligned}
$$

The full expressions for $a_{x, y n}^{*}$ and $b_{x, y n}^{*}$ are reported in the Appendix A.

The force formulations in eq. 10,12 are valid in case of zero helix angle; however, to achieve an accurate force prediction it is necessary to include such angle, therefore the equations are rearranged by including an additional summation, as proposed by Schmitz et al. [Schmitz 2006]:

$F_{x, y}(\phi)=\sum_{k=1}^{A} \sum_{j=1}^{N}\left(a_{x, y 0}^{*} \cdot d a_{p}+\sum_{n=1}^{\infty}\left(a_{x, y n}^{*} \cdot d a_{p} \cos n\left(\phi_{j}-\right.\right.\right.$ $\left.\left.\psi \cdot(k-1))+b_{x, y n}^{*} \cdot d a_{p} \sin n\left(\phi_{j}-\psi \cdot(k-1)\right)\right)\right)$

To achieve the goal, the tool is discretized in $A$ axial slices, each slice is assumed to have a zero helix angle and $d a p$ thickness. Moreover, the slices are rotated relative to one another by the angle $\psi$ :

$\psi=k_{b} d a_{p}$

$k_{b}=2 \tan \left(\alpha_{\text {hel }}\right) / D$

where $\alpha_{\text {hel }}$ is the helix angle. This assumption introduces an approximation that could lead to errors, especially when the helix angle is high and the number of slices are reduced. Moreover, an additional summation is included to the Fourier series. In this work the formulation in eq. 6 is rearranged to remove this summation and the related approximation, by introducing the integral:

$F_{x, y}(\phi)=\int_{0}^{a_{p}} \sum_{j=1}^{N}\left(a_{x, y 0}^{*} \cdot d x+\sum_{n=1}^{\infty}\left(a_{x, y n}^{*} \cdot d x \cos n\left(\phi_{j}\right.\right.\right.$

$\left.\left.\left.k_{b} x d x\right)+b_{x, y n}^{*} \cdot d x \sin n\left(\phi_{j}-k_{b} x d x\right)\right)\right)$

$F_{x, y}(\phi)=a_{x, y 0}^{*} \cdot N a_{p}+\sum_{j=1}^{N} \sum_{n=1}^{\infty}\left(\int_{0}^{a_{p}}\left(a_{x, y n}^{*} \cdot d x \cos \left(n \phi_{j}-\right.\right.\right.$

$\left.\left.n k_{b} x d x\right)+b_{x, y n}^{*} \cdot d x \sin \left(n \phi_{j}-n k_{b} x d x\right)\right)$

The phase shift $\left(k_{b} x d x\right)$ given by the helix angle can be rearranged by following simple trigonometric steps (angle sum and difference identities):

$F_{x, y}(\phi)=a_{x, y 0}^{*} \cdot N a_{p}+\sum_{j=1}^{N} \sum_{n=1}^{\infty}\left(\int_{0}^{a_{p}}\left(a_{x, y n}^{*}\right.\right.$.

$d x \cos n \phi_{j} \cos n k_{b} x d x+a_{x, y n}^{*} \cdot d x \sin n \phi_{j} \sin n k_{b} x d x+$

$b_{x, y n}^{*} \cdot d x \sin n \phi_{j} \cos n k_{b} x d x-b_{x, y n}^{*}$.

$\left.d x \sin n \phi_{j} \cos n k_{b} x d x\right)$ )

MM Science Journal | 2021 | NOVEMBER - Special Issue on HSM2021 
$F_{x, y}(\phi)=a_{x, y 0}^{*} \cdot N a_{p}+\sum_{j=1}^{N} \sum_{n=1}^{\infty}\left(\int_{0}^{a_{p}}\left(\left(a_{x, y n}^{*}\right.\right.\right.$.

$\left.d x \cos n k_{b} x d x-b_{x, y n}^{*} \cdot d x \sin n k_{b} x d x\right) \cos n \phi_{j}+\left(a_{x, y n}^{*}\right.$.

$\left.\left.\left.d x \sin n k_{b} x d x+b_{n}^{*} \cdot d x \cos n k_{b} x d x\right) \sin n \phi_{j}\right)\right)$

Solving the integral in eq. 14 , the following formulations can be derived:

$F_{x, y}(\phi)=a_{x, y 0}^{*} \cdot N a_{p}+\sum_{j=1}^{N} \sum_{n=1}^{\infty}\left[\left(\left(\frac{a_{x, y n}^{*}}{n k_{b}} \sin n k_{b} x+\right.\right.\right.$

$\left.\frac{b_{x, y n}^{*}}{n k_{b}} \cos n k_{b} x\right) \cos n \phi_{j}+\left(-\frac{a_{x, y n}^{*}}{n k_{b}} \cos n k_{b} x+\right.$

$\left.\left.\left.\frac{b_{x, y n}^{*}}{n k_{b}} \sin n k_{b} x\right) \sin n \phi_{j}\right)\right]_{0}^{a_{p}}$

$F_{x, y}(\phi)=a_{x, y 0}^{*} \cdot N a_{p}+\sum_{j=1}^{N} \sum_{n=1}^{\infty}\left(\left(\left(\frac{a_{x, y n}^{*}}{n k_{b}} \sin n k_{b} a_{p}+\right.\right.\right.$

$\left.\frac{b_{x, y n}^{*}}{n k_{b}} \cos n k_{b} a_{p}-\frac{b_{x, y n}^{*}}{n k_{b}}\right) \cos n \phi_{j}+\left(-\frac{a_{x, y n}^{*}}{n k_{b}} \cos n k_{b} a_{p}+\frac{a_{x, y n}^{*}}{n k_{b}}+\right.$

$\left.\left.\left.\frac{b_{x, y n}^{*}}{n k_{b}} \sin n k_{b} a_{p}\right) \sin n \phi_{j}\right)\right)$

The proposed expressions allow to estimate cutting forces in frequency domain in presence of helix angle using an exact expression, without the need of a specific discretization.

If run-out is neglected and teeth are evenly spaced, it is also possible to remove the summation related to the number of flutes $(\mathrm{N})$. Indeed, the formulation in equation 19 can be rewritten in:

$F_{x, y}(\phi)=a_{x, y 0}^{*} \cdot N a_{p}+N \cdot \sum_{n=N}^{\infty}\left(\left(\left(\frac{a_{x, y n}^{*}}{n k_{b}} \sin n k_{b} a_{p}+\right.\right.\right.$

$\left.\frac{b_{x, y n}^{*}}{n k_{b}} \cos n k_{b} a_{p}-\frac{b_{x, y n}^{*}}{n k_{b}}\right) \cos n \phi_{j}+\left(-\frac{a_{x, y n}^{*}}{n k_{b}} \cos n k_{b} a_{p}+\frac{a_{x, y n}^{*}}{n k_{b}}+\right.$

$\left.\left.\left.\frac{b_{x, y n}^{*}}{n k_{b}} \sin n k_{b} a_{p}\right) \sin n \phi_{j}\right)\right)$

In this case the Fourier series is not evaluated at each nmultiple of the rotational frequency but only at the ones divisible for $\mathrm{N}$, hence related only to the tooth pass frequency.

This additional simplification allows to analytically predict the Fast Fourier Transform (FFT) components of the cutting forces in $\mathrm{x}$ and $\mathrm{y}$ directions as follows:

- For the constant term (zero frequency):

$a_{x, y 0}^{*} \cdot N a_{p}$

- For tooth pass frequency and its harmonics:

$\frac{1}{2} N\left(\left(\frac{a_{x, y n}^{*}}{n k_{b}} \sin \left(n k_{b} a_{p}\right)+\frac{b_{x, y n}^{*}}{n k_{b}} \cos \left(n k_{b} a_{p}\right)-\frac{b_{x, y n}^{*}}{n k_{b}}\right)-\right.$

$\left.i\left(-\frac{a_{x, y n}^{*}}{n k_{b}} \cos \left(n k_{b} a_{p}\right)+\frac{a_{x, y n}^{*}}{n k_{b}}+\frac{b_{x, y n}^{*}}{n k_{b}} \sin \left(n k_{b} a_{p}\right)\right)\right)$

where $n$ is multiple of the number of flutes $(N)$.

It is interesting to point out that, in analogy of what proposed for force shape characteristics [Yang 2005], two main angles are responsible for the frequency content of the cutting forces:

- the radial engagement angle $\left(\alpha_{e n}\right)$ responsible for $\phi_{i n-}$

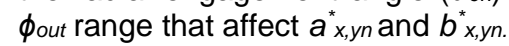

- the axial engagement angle or sweep angle $\left(\alpha_{s w}\right)$ that depends on helix angle and axial depth of cut according with the following equation:

$\alpha_{s w}=k_{b} a_{p}$

\section{DEPTH OF CUT MONITORING}

In this work an application of the developed formulations for cutting force frequency content is presented. This is a first step for the development of an algorithm able to detect an unwanted rise of depths of cut during the milling process (e.g., bad programming, collision). The system should be able to monitor the process by means of cutting force measurement with the following features:

- Requiring the smallest number of parameters, and only the ones known by the operator.

- Not requiring information about cutting force model coefficients (i.e., $\mathrm{K}_{\mathrm{tc}}, \mathrm{K}_{\mathrm{rc}} \mathrm{K}_{\mathrm{te}}, \mathrm{K}_{\mathrm{re}}$ ).

- Not requiring information about cutting direction.

To achieve such ambitious goal, the idea is to adopt total cutting force on $\mathrm{x}$-y plane $\left(F_{\text {tot }}\right)$ that can be computed as:

$F_{t o t}(\phi)=\sqrt{F_{t}^{2}(\phi)+F_{r}^{2}(\phi)}=\sqrt{F_{x}^{2}(\phi)+F_{y}^{2}(\phi)}$

This cutting force can be easily acquired during the process without the need of knowing the cutter direction in $x-y$ plane. Moreover, it is possible to demonstrate that in the frequency domain, in analogy with $\mathrm{x}$ and $\mathrm{y}$ direction forces, $F_{\text {tot }}$ can be written as:

$$
\begin{aligned}
& F_{\text {tot }}(\phi)=a_{\text {tot } 0}^{*} \cdot N a_{p}+N \cdot \sum_{n=N}^{\infty}\left(\left(\left(\frac{a_{\text {totn }}^{*}}{n k_{b}} \sin n k_{b} a_{p}+\right.\right.\right. \\
& \left.\frac{b_{\text {totn }}^{*}}{n k_{b}} \cos n k_{b} a_{p}-\frac{b_{\text {totn }}^{*}}{n k_{b}}\right) \cos n \phi_{j}+\left(-\frac{a_{\text {totn }}^{*}}{n k_{b}} \cos n k_{b} a_{p}+\frac{a_{\text {totn }}^{*}}{n k_{b}}+\right. \\
& \left.\left.\left.\frac{b_{\text {totn }}^{*}}{n k_{b}} \sin n k_{b} a_{p}\right) \sin n \phi_{j}\right)\right)
\end{aligned}
$$

In case of lumped shear cutting force model (i.e., $K t e=K r e=0)$, the formulation for $a^{*}$ totn and $b^{*}$ totn can be written as:

$$
\begin{aligned}
& a_{\text {tot } 0}^{*}=a_{t o t 0}^{* *} \cdot f_{z} \sqrt{{K_{t c}{ }^{2}+K_{r c}{ }^{2}}^{2}} \\
& a_{\text {totn }}^{*}=a_{t o n}^{* *} \cdot f_{z} \sqrt{{K_{t c}{ }^{2}+K_{r c}{ }^{2}}^{2}} \\
& b_{\text {ton }}^{*}=b_{\text {ton }}^{* *} \cdot f_{z} \sqrt{{K_{t c}{ }^{2}+K_{r c}{ }^{2}}^{2}}
\end{aligned}
$$

where the full expressions for $a^{* \star}$ totn and $b^{* *}$ totn are reported in the Appendix A. Using expressions in eq. 29-31, eq. 28 $F_{\text {tot }}$ can be written as:

$F_{\text {tot }}(\phi)=a_{\text {toto }}^{* *} \cdot f_{z} \sqrt{{K_{t c}{ }^{2}+K_{r c}{ }^{2}}_{2}} \cdot N a_{p}+N \cdot f_{z} \sqrt{{K_{t c}{ }^{2}+K_{r c}{ }^{2}}^{2}}$.

$\sum_{n=N}^{\infty}\left(\left(\left(\frac{a_{\text {totn }}^{* *}}{n k_{b}} \sin n k_{b} a_{p}+\frac{b_{t o t n}^{* *}}{n k_{b}} \cos n k_{b} a_{p}-\frac{b_{t o t n}^{* *}}{n k_{b}}\right) \cos n \phi_{j}+\right.\right.$

$\left.\left.\left(-\frac{a_{t o t n}^{* *}}{n k_{b}} \cos n k_{b} a_{p}+\frac{a_{\text {totn }}^{* *}}{n k_{b}}+\frac{b_{t o t n}^{* *}}{n k_{b}} \sin n k_{b} a_{p}\right) \sin n \phi_{j}\right)\right)$

FFT components for $F_{\text {tot }}$ using lumped shear cutting force model are:

- For the constant term (zero frequency):

$A_{0}=a_{t o t 0}^{* *} \cdot f_{z} \sqrt{K_{t c}{ }^{2}+K_{r c}^{2}} \cdot N a_{p}$

- For tooth pass frequency and its harmonics:

$C_{n}=\frac{1}{2} N \cdot f_{z} \sqrt{K_{t c}{ }^{2}+K_{r c}^{2}} \cdot\left(\left(\frac{a_{t o t n}^{* *}}{n k_{b}} \sin \left(n k_{b} a_{p}\right)+\right.\right.$

$\left.\frac{b_{t o t n}^{* *}}{n k_{b}} \cos \left(n k_{b} a_{p}\right)-\frac{b_{x, y n}^{*}}{n k_{b}}\right)-i\left(-\frac{a_{t o t n}^{* *}}{n k_{b}} \cos \left(n k_{b} a_{p}\right)+\frac{a_{t o t n}^{* *}}{n k_{b}}+\right.$

$\left.\left.\frac{b_{t o t n}^{* *}}{n k_{b}} \sin \left(n k_{b} a_{p}\right)\right)\right) n=2,3, \ldots, \infty$

Based on these formulations, it is possible to propose a monitoring solution based on $V_{n}$, the ratio between magnitude of $C_{n}$ and $A_{0}$, that does not require the knowledge of any cutting force coefficients.

$V_{n}=\left|C_{n}\right| / A_{o}$ 
The error between predicted value and measured value could be expressed as:

$f_{o}=\frac{\left\|V_{n p}-V_{n m}\right\|^{2}}{\left\|V_{n m}\right\|^{2}} n=2,3, \ldots, c$

where $c$ is the number of Fourier coefficients considered, $V_{n p}$ and $V_{n m}$ are the predicted and measured values of the ratio formulated in eq. 35. and the symbol "II" indicates the 2 -norm of the vector. The proposed monitoring method should be organized following these steps:

1) Cutting forces are acquired in time domain on different directions with adequate sampling frequency.

2) $x$-y plane total force is computed by using eq. 6 .

3) A procedure to remove the influence of run-out as the one proposed by Rubeo et al. [Rubeo 2016] is applied.

4) Discrete Fast Fourier Transformation (DFFT) of the signal is performed and constant term $A$ om and magnitude of the tooth pass frequency $\left(C_{n m}\right)$ and harmonics are isolated.

5) Error between predicted and experimental results, is computed according to eq. 36 .

The computed error could be used to monitor the process or as an error function to estimate depths of cut $\left(a_{r}\right.$ and $\left.a_{p}\right)$ from experimental data via optimization algorithm.

\section{NUMERICAL VALIDATION}

The formulations presented in section 2 were tested using a numerical validation. A series of time-domain simulations were carried out using the cutting force model in eq. 1-7. Discrete Fast Fourier Transformation (DFFT) was then applied to simulated cutting forces in feed ( $x$ ) and crossfeed $(y)$ directions to compute the spectra of the cutting forces in frequency domain. The derived DFFT of the forces were then compared with the analytical formulations proposed. In addition, the proposed depth of cut monitoring solution is applied to the signals. This procedure allows to test the proposed solution in a simulative environment using a dual mechanism force model compared to the lumped shear force model adopted to define the monitoring approach.

First, $f_{0}$ is computed with the simulated depths of cut, comparing analytically predicted frequency content ratio $\left(V_{n p}\right)$ with measured ratios $\left(V_{n m}\right)$. Then, a simple optimization was implemented to calculate the best depths of cut $\left(a_{p}\right.$ and $\left.a_{r}\right)$ to minimize the $f_{o}$ error.

Several tests were performed to analyze the force spectrum in different conditions, with the same spindle speed and feed per tooth, $6366 \mathrm{rpm}$ and $0.1 \mathrm{~mm}$ respectively. The first simulation considers a 4-fluted $12 \mathrm{~mm}$ end-mill with $45^{\circ}$ helix angle engaged $3 \mathrm{~mm}$ axially $\left(a_{p}\right)$ and $4 \mathrm{~mm}$ in the radial direction $\left(\mathrm{a}_{\mathrm{r}}\right)$.

Cutting parameters used in the different simulations are summarized in Tab. 1, along with the results of the monitoring solution (coefficients considered $c=10$ ). Cutting force coefficients adopted, typical of an aluminum [Grossi 2017], are reported in Tab. 2. The results of the first test are reported in Fig. 1, where magnitude of the FFT is shown.

Using the formulation in section 2 spectrum of forces on both directions is accurately predicted, confirming the validity of the proposed formulations. Moreover, using the simplified formulations presented in section 3 for the monitoring approach, resulting error $\left(f_{0}\right)$ between predicted and numerical values is very low, even of applied to cutting forces simulated considering edge coefficients.
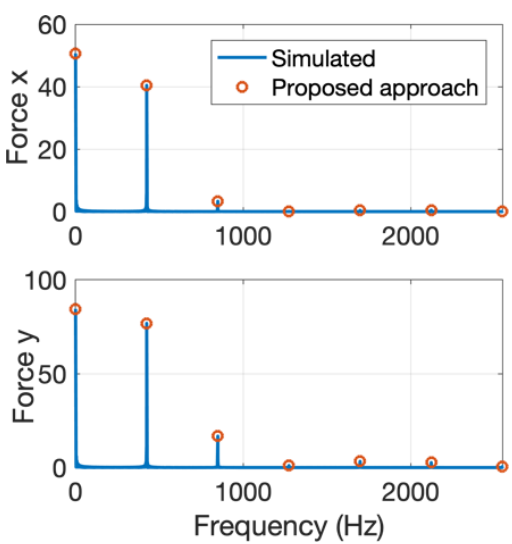

Fig. 1: Cutting forces FFT magnitude $(\mathrm{N})$, test 1

Tab. 1: Cutting conditions tested (spindle speed $6366 \mathrm{rpm}$ and $0.1 \mathrm{~mm}$ feed per tooth)

\begin{tabular}{ccccccccc}
\hline ID & $\begin{array}{l}\mathrm{a}_{\mathrm{p}} \\
(\mathrm{mm})\end{array}$ & $\begin{array}{l}\mathrm{a}_{\mathrm{r}} \\
(\mathrm{mm})\end{array}$ & $\mathrm{N}$ & $\begin{array}{l}\alpha_{\text {hel }} \\
\left({ }^{\circ}\right)\end{array}$ & $\begin{array}{l}\mathrm{D} \\
(\mathrm{mm})\end{array}$ & $f_{o}(\%)$ & $\begin{array}{l}\mathrm{a}_{\mathrm{p}}^{\mathrm{opt}} \\
(\mathrm{mm})\end{array}$ & $\begin{array}{l}\mathrm{a}_{\mathrm{r}}^{\mathrm{opt}} \\
(\mathrm{mm})\end{array}$ \\
\hline 1 & 3 & 4 & 4 & 45 & 12 & 0.03 & 3.00 & 4.07 \\
2 & 3 & 4 & 2 & 45 & 12 & 0.01 & 3.00 & 4.07 \\
3 & 3 & 4 & 6 & 45 & 12 & 0.03 & 3.00 & 4.05 \\
4 & 3 & 4 & 4 & 25 & 12 & 0.03 & 3.00 & 4.07 \\
5 & 3 & 4 & 4 & 50 & 12 & 0.03 & 3.00 & 4.07 \\
6 & 1 & 4 & 4 & 45 & 12 & 0.02 & 1.00 & 4.07 \\
7 & 9 & 4 & 4 & 45 & 12 & 0.27 & 9.01 & 4.07 \\
8 & 3 & 2 & 4 & 45 & 12 & 0.02 & 3.00 & 2.06 \\
9 & 3 & 10 & 4 & 45 & 12 & 0.02 & 2.97 & 10.07 \\
10 & 8.24 & 4 & 4 & 20 & 12 & 0.03 & 8.24 & 4.07 \\
11 & 6.67 & 4 & 4 & 45 & 20 & 0.02 & 6.67 & 4.11 \\
\hline
\end{tabular}

Tab. 2: Cutting force coefficients.

\begin{tabular}{llll}
\hline $\mathrm{K}_{\mathrm{tc}}(\mathrm{MPa})$ & $\mathrm{K}_{\mathrm{te}}(\mathrm{N} / \mathrm{mm})$ & $\mathrm{K}_{\mathrm{rc}}(\mathrm{MPa})$ & $\mathrm{K}_{\mathrm{re}}(\mathrm{N} / \mathrm{mm})$ \\
\hline 770 & 0.5 & 150 & 2 \\
\hline
\end{tabular}

Applying depth of cut identification algorithm based on $f_{o}$ on the signals (without knowledge of cutting force coefficients and depths of cut), computed depths of cut (Tab. 1) are close to the ones imposed, with a small increase in radial depth of cut $(4.07 \mathrm{~mm})$. Indeed, the radial depth of cut influences differently the frequency content of the signal in presence of edge coefficients (see appendix A). Similar trend can be found for all the numerical results.

In Fig. 2 the influence of the number of flutes of the tool is highlighted. As expected, increasing the number of flutes from 2 to 6 , reductions on significant harmonics are found. Indeed, increasing $\mathrm{n}$, the Fourier coefficients reduce their value (as clear from the equations in appendix $A$ ), and this apply also to $\mathrm{N}$ since it influences the coefficients number to be computed.

Fig. 2 confirms the accuracy of the proposed formulations and the same trend in the monitoring solution application. This is valid also for the results shown in Fig. 3, where helix angle of the tool is changed.

Helix angle influences tooth pass frequency and harmonics indeed, looking at the proposed formulations, it affects $\alpha_{s w}$ and hence the shape of the force. As the helix angle increases, the force is smoother reducing the relevance of harmonics. 

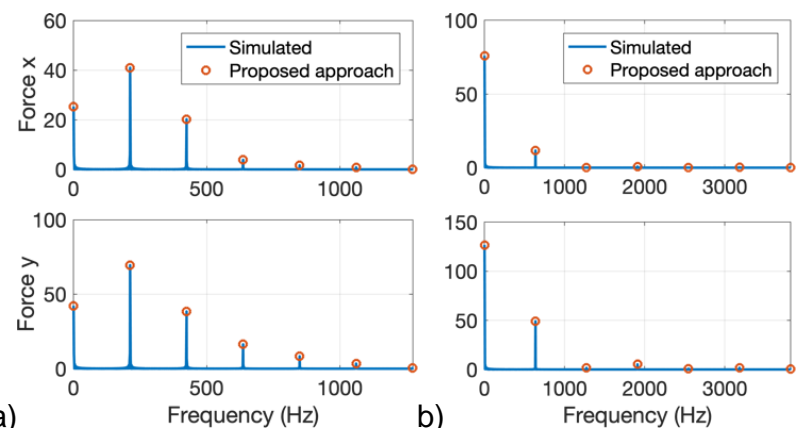

Fig. 2: Effect of flutes number a) $N=2, b) \mathrm{N}=6$
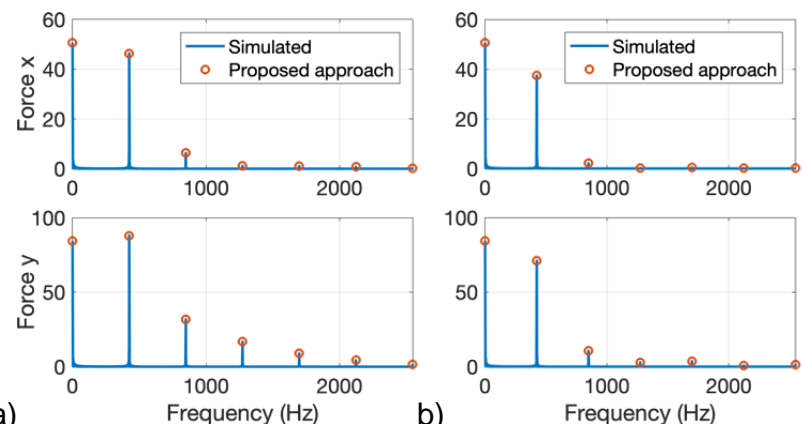

Fig. 3: Effect of helix angle $\left(\alpha_{\text {hell }}\right.$ a) $25^{\circ}$, b) $50^{\circ}$

In Fig. 4 the effect of axial depth of cut is shown. Despite high axial depth of cut $(9 \mathrm{~mm})$ the error $f_{o}$ is higher (even if lower than $0.3 \%$ ), and the depths of cut estimated via optimization algorithm are still close to the simulated ones. For what concern the effect of axial depth of cut, an increase of the value makes the constant term rise, and therefore it reduces the relevance of the tooth pass frequency and its harmonics.

Similar considerations can be drawn for radial depth of cut in the case of cross-feed force (Fig. 5). On the contrary, feed force presents a different trend, indeed when the radial depth of cut reaches a value close to the full-immersion tooth pass frequency becomes relevant. In addition to the analysis of the effects of the different parameters on the cutting forces spectra, two simulations were performed keeping constant the two engagement angles $\left(\alpha_{e n}, \alpha_{s w}\right)$, equal to the one of test 1 (Fig. 6).

This is achieved by changing depths of cut and tool characteristics.
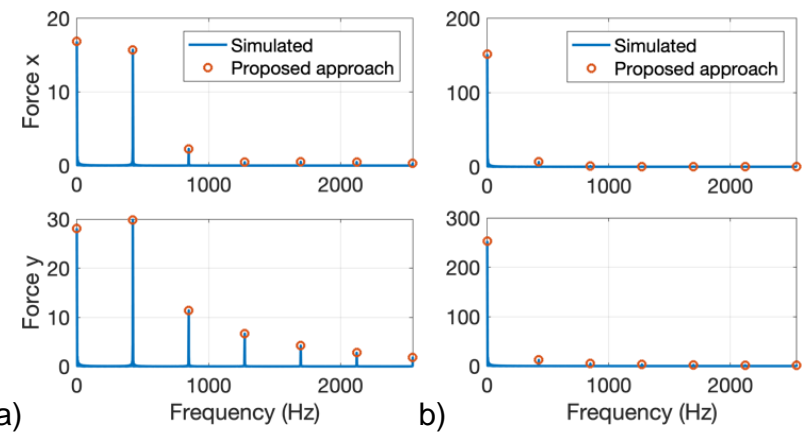

a)

Fig. 4: Effect of axial depth of cut (ap) a) $1 \mathrm{~mm}$, b) $9 \mathrm{~mm}$
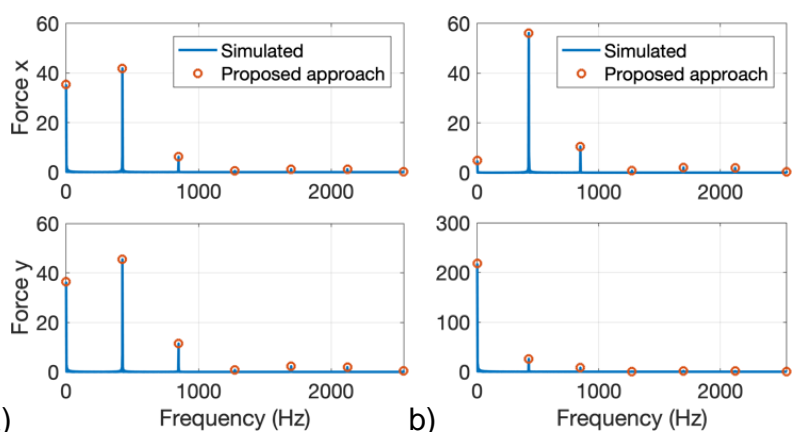

Fig. 5: Effect of radial depth of cut (ar) a) $2 \mathrm{~mm}$, b) $10 \mathrm{~mm}$
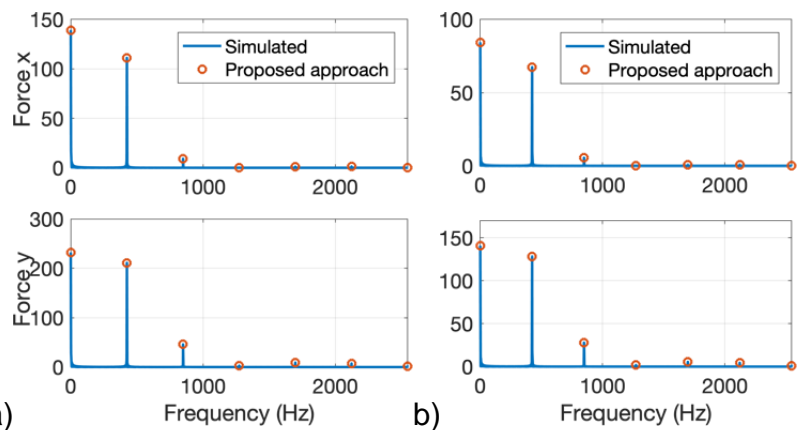

Fig. 6: Conditions with the same $\alpha_{\text {en }}$ and $\alpha_{s w}$

Results show that the spectra of the forces of test 10,11 and 1 present the same distribution and the same ratios between the different components, even if the values change. This confirm that the engagement angles are important for the shape of the cutting forces.

All the simulated tests show a low error $\left(f_{0}\right)$ and identification algorithm, based on the formulation presented in section 3 , returns a fair estimation of depths of cut.

\section{EXPERIMENTAL APPLICATION}

In addition to the numerical validation, the monitoring solution idea proposed in section 3 was applied to cutting force data, experimentally acquired.

Two cutting tests were performed on a DMG MORI DMU 75 milling machine on Alluminum (6082-T4) using a four-fluted end-mill Garant with $12 \mathrm{~mm}$ diameter and $45^{\circ}$ helix angle.

During the tests, a Kistler 9257A table dynamometer was used to measure forces. Spindle speed was set to $6366 \mathrm{rpm}$ and feed per tooth to $0.1 \mathrm{~mm}$, the other cutting parameters adopted are summarized in Tab. 3. The proposed method was applied to the measured forces, as presented in section 4 for the simulated ones.

Tab. 3: Cutting conditions tested (spindle speed $6366 \mathrm{rpm}$ and $0.1 \mathrm{~mm}$ feed per tooth)

\begin{tabular}{ccccccccc}
\hline ID & $\begin{array}{l}\mathrm{a}_{\mathrm{p}} \\
(\mathrm{mm})\end{array}$ & $\begin{array}{l}\mathrm{a}_{\mathrm{r}} \\
(\mathrm{mm})\end{array}$ & $\begin{array}{c}\mathrm{N} \\
\alpha_{\text {hel }} \\
\left({ }^{\circ}\right)\end{array}$ & $\begin{array}{l}\mathrm{D} \\
(\mathrm{mm})\end{array}$ & $f_{o}(\%)$ & $\begin{array}{l}\mathrm{a}_{\mathrm{p}}{ }^{\mathrm{opt}} \\
(\mathrm{mm})\end{array}$ & $\begin{array}{l}\mathrm{a}_{\mathrm{r}}^{\mathrm{opt}} \\
(\mathrm{mm})\end{array}$ \\
\hline $\mathrm{A}$ & 15 & 2.5 & 4 & 45 & 12 & 0.59 & 13.4 & 2.84 \\
$\mathrm{~B}$ & 7 & 1 & 4 & 45 & 12 & 0.97 & 6.77 & 1.15 \\
\hline
\end{tabular}




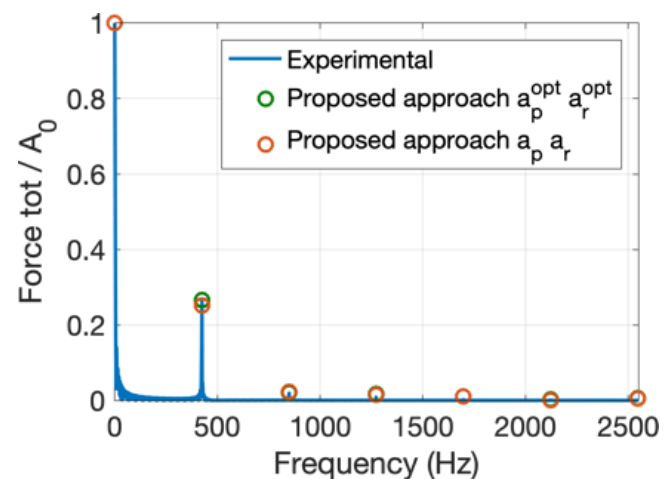

Fig. 7: FFT of the measured total force for test $A\left(a_{p}=15\right.$ $\mathrm{mm}, \mathrm{a}_{r}=2.5 \mathrm{~mm}$ )

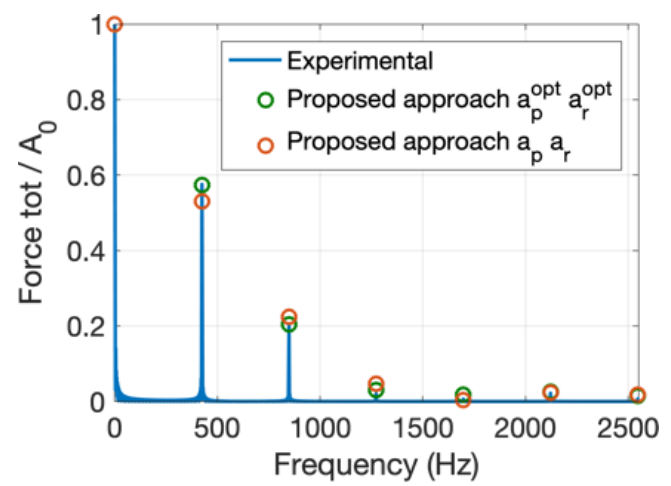

Fig. 8: FFT of the measured total force for test $B\left(a_{p}=7\right.$ $\mathrm{mm}, \mathrm{a}_{\mathrm{r}}=1 \mathrm{~mm}$ )

The acquired cutting forces were post-processed off-line following the steps presented in section 3 . These forces were compensated to reduce the distortions derived by the system dynamics using the approach proposed by Scippa et al. [Scippa 2015], and post-processed to compensate tool run-out as adopted by Rubeo et al. [Rubeo 2016].

Since the focus of the proposed monitoring idea is based on the total force (i.e., resultant of the tangential and radial forces), such force was computed by combining $\mathrm{X}$-force and $\mathrm{Y}$-force.

Comparisons between measured force spectra (normalized to the constant force) and the proposed formulations using the imposed depths of cut and the ones obtained by the optimization algorithm are presented Fig. 7 and Fig. 8.

The application to the two experimental tests returns fair accuracy of the proposed method. Indeed, as shown in Tab. 3 , in both cases $f_{0}$ is low (less than $1 \%$ ), and the estimated depths of cut are close to the ones imposed.

Although these preliminary results are promising, the results are not good enough to consider the proposed method, as implement here, able to be used to estimate accurately the depths of cut. However, the system should be already able to detect a big change in engagement conditions, starting by the expected depths of cut by using the formulated $f_{o}$ error.

\section{CONCLUSION}

Cutting forces are one of the most significant signals for monitor milling process. Indeed, it presents peculiar characteristics that depend on several aspects, such as tool wear, cutting parameters, and affect different process outcomes, such as machining error or vibrations.
In this work, the frequency content of the cutting forces (i.e., frequency spectrum) is analyzed and analytical formulations are proposed with the aim of developing a monitoring solution for depths of cut identification. Indeed, the real-time knowledge of both axial and radial depth of cut could be very useful in actual machining operation to prevent the tool from working with unwanted parameters, leading to aggressive tool wear or failures.

Investigating the frequency content of the cutting forces, the total force normalized spectrum respect to the constant term seems to be the most promising parameter to be used for depths of cut monitoring. Indeed, using a lumped shear force model, the proposed normalized spectrum is independent on the cutting force coefficients and feed per tooth. This allows to develop an identification algorithm without the need of prior knowledge of cutting force coefficients and cutting directions, making it more suitable for an industrial implementation.

The proposed analytical formulations for feed and crossfeed cutting force spectra have been numerically investigated, confirming their accuracy. Moreover, a preliminary implementation of the monitoring solution is applied to simulated and experimental cutting forces. Although the method seems to be promising, the results show that, as implemented in this work, the accuracy is not enough to precisely identify the depths of cut. However, the proposed error formulation could be already used to detect big changes in engagement conditions, allowing to intervene in case of error in the programming or malfunctioning (e.g., tool poor clamping).

The proposed approach needs to be improved in the implementation (e.g., measurement post-processing, realtime) and further investigations must be carried out on the analytical formulations to obtain a robust approach. A possible solution could rely on more than one parameter, that could be based not only on cutting force frequency content but also on force shape in time-domain.

\section{REFERENCES}

[Altintas and Budak 1995] Altintas, Y. and Budak, E. Analytical Prediction of Stability Lobes in Milling. CIRP Annals - Manufacturing Technology, 1995, Vol. 44, No.1, pp. 357-362. doi: http://dx.doi.org/10.1016/S00078506(07)62342-7.

[Altintas and Yellowley 1987] Altintas, Y. and Yellowley, I. The identification of radial width and axial depth of cut in peripheral milling. International Journal of Machine Tools and Manufacture, 1987, Vol. 27, No.3, pp. 367-381. doi: https://doi.org/10.1016/S0890-6955(87)80010-X.

[Bachrathy Munoa and Stepan 2016] Bachrathy, D., Munoa, J., et al. Experimental validation of appropriate axial immersions for helical mills. International Journal of Advanced Manufacturing Technology, 2016, Vol. 84, No.58, pp. 1295-1302. doi: 10.1007/s00170-015-7748-0.

[Budak 2003] Budak, E. An analytical design method for milling cutters with nonconstant pitch to increase stability, Part 2: Application. Journal of Manufacturing Science and Engineering, Transactions of the ASME, 2003, Vol. 125, No.1, pp. 35-38. doi: 10.1115/1.1536656.

[Budak Altintas and Armarego 1996] Budak, E., Altintas, Y., et al. Prediction of Milling Force Coefficients From Orthogonal Cutting Data. Journal of Manufacturing Science 
and Engineering, 1996, Vol. 118, No.2, pp. 216-224. doi: 10.1115/1.2831014.

[Campatelli and Scippa 2012] Campatelli, G. and Scippa, A. Prediction of milling cutting force coefficients for aluminum 6082-T4. Procedia CIRP, 2012, Vol. 1, No.1, pp. 563-568. doi: 10.1016/j.procir.2012.04.100.

[Choi and Yang 1999] Choi, J. G. and Yang, M. Y. Inprocess prediction of cutting depths in end milling. International Journal of Machine Tools and Manufacture, 1999, Vol. 39, No.5, pp. 705-721. doi: 10.1016/S08906955(98)00067-4.

[Ducroux Fromentin Viprey Prat and D'Acunto 2021] Ducroux, E., Fromentin, G., et al. New mechanistic cutting force model for milling additive manufactured Inconel 718 considering effects of tool wear evolution and actual tool geometry. Journal of Manufacturing Processes, 2021, Vol. 64, No.January, pp. 67-80. doi: 10.1016/j.jmapro.2020.12.042.

[Grossi 2017] Grossi, N. Accurate and fast measurement of specific cutting force coefficients changing with spindle speed. International Journal of Precision Engineering and Manufacturing, 2017, Vol. 18, No.8. doi: 10.1007/s12541017-0137-x.

[Grossi Sallese Scippa and Campatelli 2017] Grossi, N., Sallese, L., et al. Improved experimental-analytical approach to compute speed-varying tool-tip FRF. Precision Engineering, 2017, Vol. 48, . doi: 10.1016/j.precisioneng.2016.11.011.

[Grossi Scippa Croppi Morelli and Campatelli 2019] Grossi, N., Scippa, A., et al. Adaptive toolpath for 3-axis milling of thin walled parts. MM Science Journal, 2019, Vol. 2019, No.November, pp. 3378-3385. doi: 10.17973/MMSJ.2019_11_2019096.

[Junz Wang and Zheng 2002] Junz Wang, J.-J. and Zheng, C. M. An analytical force model with shearing and ploughing mechanisms for end milling. International Journal of Machine Tools and Manufacture, 2002, Vol. 42, No.7, pp. 761-771. doi: https://doi.org/10.1016/S08906955(02)00019-6.

[Kapoor Ehmann Kapoor DeVor and Lazoglu 1997] Kapoor, S. G., Ehmann, K. F., et al. Machining Process Modeling: A Review. Journal of Manufacturing Science and Engineering, 1997, Vol. 119, No.4B, pp. 655-663. doi: 10.1115/1.2836805.

[Kiran and Kayacan 2019] Kiran, K. and Kayacan, M. C. Cutting force modeling and accurate measurement in milling of flexible workpieces. Mechanical Systems and Signal Processing, 2019, Vol. 133, , p. 106284. doi: 10.1016/j.ymssp.2019.106284.

[Leal-Munoz Diez Perez and Vizan 2018] Leal-Munoz, E., Diez, E., et al. Identification of the Actual Process Parameters for Finishing Operations in Peripheral Milling. Journal of Manufacturing Science and Engineering, Transactions of the ASME, 2018, Vol. 140, No.8, pp. 1-7. doi: $10.1115 / 1.4039917$.

[Liu Cheng Webb and Luo 2002] Liu, X. W., Cheng, K., et al. Prediction of cutting force distribution and its influence on dimensional accuracy in peripheral milling. International Journal of Machine Tools and Manufacture, 2002, Vol. 42, No.7, pp. 791-800. doi: 10.1016/S0890-6955(02)00016-0.

[Matsumura and Tamura 2017] Matsumura, T. and Tamura, S. Cutting Force Model in Milling with Cutter Runout. Procedia CIRP, 2017, Vol. 58, , pp. 566-571. doi: 10.1016/j.procir.2017.03.268.

[Nishida Okumura Sato and Shirase 2018] Nishida, I., Okumura, R., et al. Cutting force and finish surface simulation of end milling operation in consideration of static tool deflection by using voxel model. Procedia CIRP, 2018, Vol. 77, , pp. 574-577. doi: 10.1016/j.procir.2018.08.218.

[Rubeo and Schmitz 2016] Rubeo, M. A. and Schmitz, T. T. L. Mechanistic force model coefficients: A comparison of linear regression and nonlinear optimization. Precision Engineering, 2016, Vol. 45, , pp. 311-321. doi: 10.1016/j.precisioneng.2016.03.008.

[Schmitz and Mann 2006] Schmitz, T. L. and Mann, B. P. Closed-form solutions for surface location error in milling. International Journal of Machine Tools and Manufacture, 2006, Vol. 46, No.12-13, pp. 1369-1377. doi: http://dx.doi.org/10.1016/j.jmachtools.2005.10.007.

[Scippa Sallese Grossi and Campatelli 2015] Scippa, A., Sallese, L., et al. Improved dynamic compensation for accurate cutting force measurements in milling applications. Mechanical Systems and Signal Processing, 2015, Vol. 54, , pp. 314-324. doi: 10.1016/j.ymssp.2014.08.019.

[Wang and Zheng 2003] Wang, J. J. J. and Zheng, C. M. Identification of cutter offset in end milling without a prior knowledge of cutting coefficients. International Journal of Machine Tools and Manufacture, 2003, Vol. 43, No.7, pp. 687-697. doi: 10.1016/S0890-6955(03)00028-2.

[Yang DeVor and Kapoor 2004] Yang, L., DeVor, R. E., et al. Analysis of Force Shape Characteristics and Detection of Depth-of-Cut Variations in End Milling. Journal of Manufacturing Science and Engineering, 2004, Vol. 127, No.3, pp. 454-462. doi: 10.1115/1.1947207.

[Yang DeVor and Kapoor 2005] Yang, L., DeVor, R. E., et al. Analysis of force shape characteristics and detection of depth-of-cut variations in end milling. Journal of Manufacturing Science and Engineering, Transactions of the ASME, 2005, Vol. 127, No.3, pp. 454-462. doi: 10.1115/1.1947207. 


\section{APPENDIX A}

The coefficients for the Fourier series computation of cutting forces are provided here.

Coefficients for cutting force in the feed direction $(x)$ considering edge coefficients

$a_{x 0}^{*}=-\frac{1}{2 \pi}\left[-K_{t c} f_{z}\left(\frac{1}{4} \cos (2 x)\right)-K_{r c} f_{z}\left(-\frac{x}{2}+\frac{1}{4} \sin (2 x)\right)+K_{t e} \sin (x)-K_{r e} \cos (x)\right]_{\phi_{\text {in }}}^{\phi_{\text {out }}}$

$a_{x 1}^{*}=-\frac{1}{\pi}\left[K_{t c} f_{z}\left(-\frac{1}{4} \cos (x)-\frac{1}{12} \cos (3 x)\right)-K_{r c} f_{z}\left(-\frac{1}{4} \sin (x)+\frac{1}{12} \sin (3 x)\right)+K_{t e}\left(\frac{1}{2} x+\frac{1}{4} \sin (2 x)\right)-K_{r e}\left(\frac{1}{4} \cos (2 x)\right)\right]_{\phi_{\text {in }}}^{\phi_{\text {out }}(38)}$

$a_{x 2}^{*}=-\frac{1}{\pi}\left[-K_{t c} f_{z}\left(\frac{1}{16} \cos (4 x)\right)-K_{r c} f_{z}\left(\frac{1}{4} x-\frac{1}{4} \sin (2 x)+\frac{1}{16} \sin (4 x)\right)+K_{t e}\left(\frac{1}{2} \sin (x)+\frac{1}{6} \sin (3 x)\right)-K_{r e}\left(-\frac{1}{2} \cos (x)+\right.\right.$ $\left.\left.\frac{1}{6} \cos (3 x)\right)\right]_{\phi_{\text {in }}}^{\phi_{\text {out }}}$

$a_{x j}^{*}=-\frac{1}{\pi}\left[K_{t c} f_{z}\left(\frac{1}{4(j-2)} \cos ((j-2) x)-\frac{1}{4(j+2)} \cos ((j+2) x)\right)-K_{r c} f_{z}\left(-\frac{1}{2 j} \sin (j x)+\frac{1}{4(j-2)} \sin ((j-2) x)+\frac{1}{4(j+2)} \sin ((j+\right.\right.$

$\left.2) x))+K_{t e}\left(\frac{1}{2(j-1)} \sin ((j-1) x)+\frac{1}{2(j+1)} \sin ((j+1) x)\right)-K_{r e}\left(-\frac{1}{2(j-1)} \cos ((j-1) x)+\frac{1}{2(j+1)} \cos ((j+1) x)\right)\right]_{\phi_{\text {in }}}^{\phi_{\text {out }}}$

$b_{x 1}^{*}=-\frac{1}{\pi}\left[K_{t c} f_{z}\left(\frac{1}{4} \sin (x)-\frac{1}{12} \sin (3 x)\right)-K_{r c} f_{z}\left(\frac{3}{4} \cos (x)-\frac{1}{12} \cos (3 x)\right)+K_{t e}\left(-\frac{1}{4} \cos (2 x)\right)-K_{r e}\left(-\frac{1}{2} x+\frac{1}{4} \sin (2 x)\right)\right]_{\phi_{\text {in }}}^{\phi_{\text {out }}}(41)$

$b_{x 2}^{*}=-\frac{1}{\pi}\left[K_{t c} f_{z}\left(\frac{1}{4} x-\frac{1}{16} \sin (4 x)\right)-K_{r c} f f_{z}\left(\frac{1}{4} \cos (2 x)-\frac{1}{16} \cos (4 x)\right)+K_{t e}\left(-\frac{1}{2} \cos (x)-\frac{1}{6} \cos (3 x)\right)-K_{r e}\left(-\frac{1}{2} \sin (x)+\right.\right.$

$\left.\left.\frac{1}{6} \sin (3 x)\right)\right]_{\phi_{\text {in }}}^{\phi_{\text {out }}}$

$b_{x j}^{*}=-\frac{1}{\pi}\left[K_{t c} f_{z}\left(\frac{1}{4(j-2)} \sin ((j-2) x)-\frac{1}{4(j+2)} \sin ((j+2) x)\right)-K_{r c} f_{z}\left(\frac{1}{2 j} \cos (j x)-\frac{1}{4(j-2)} \cos ((j-2) x)-\frac{1}{4(j+2)} \cos ((j+\right.\right.$

$\left.2) x))+K_{t e}\left(-\frac{1}{2(j-1)} \cos ((j-1) x)-\frac{1}{2(j+1)} \cos ((j+1) x)\right)-K_{r e}\left(-\frac{1}{2(j-1)} \sin ((j-1) x)+\frac{1}{2(j+1)} \sin ((j+1) x)\right)\right]_{\phi_{\text {in }}}^{\phi_{\text {out }}}$

Coefficients for cutting force in the cross-feed $(y)$ considering edge coefficients

$a_{y 0}^{*}=-\frac{1}{2 \pi}\left[K_{t c} f_{z}\left(-\frac{x}{2}+\frac{1}{4} \sin (2 x)\right)-K_{r c} f_{z}\left(\frac{1}{4} \cos (2 x)\right)+K_{t e} \cos (x)+K_{r e} \sin (x)\right]_{\phi_{\text {in }}}^{\phi_{\text {out }}}$

$a_{y 1}^{*}=-\frac{1}{\pi}\left[K_{t c} f_{z}\left(-\frac{1}{4} \sin (x)+\frac{1}{12} \sin (3 x)\right)+K_{r c} f_{z}\left(-\frac{1}{4} \cos (x)-\frac{1}{12} \cos (3 x)\right)+K_{t e}\left(\frac{1}{4} \cos (2 x)\right)+K_{r e}\left(\frac{1}{2} x+\frac{1}{4} \sin (2 x)\right]_{\phi_{\text {out }}}^{\phi_{\text {out }}}(45)\right.$

$a_{y 2}^{*}=-\frac{1}{\pi}\left[K_{t c} f_{z}\left(\frac{1}{4} x-\frac{1}{4} \sin (2 x)+\frac{1}{16} \sin (4 x)\right)-K_{r c} f_{z}\left(\frac{1}{16} \cos (4 x)\right)+K_{t e}\left(-\frac{1}{2} \cos (x)+\frac{1}{6} \cos (3 x)\right)+K_{r e}\left(\frac{1}{2} \sin (x)+\right.\right.$ $\left.\frac{1}{6} \sin (3 x)\right]_{\phi_{\text {in }}}^{\phi_{\text {out }}}$

$a_{y j}^{*}=-\frac{1}{\pi}\left[K_{t c} f_{z}\left(-\frac{1}{2 j} \sin (j x)+\frac{1}{4(j-2)} \sin ((j-2) x)+\frac{1}{4(j+2)} \sin ((j+2) x)\right)+K_{r c} f_{z}\left(\frac{1}{4(j-2)} \cos ((j-2) x)-\frac{1}{4(j+2)} \cos ((j+\right.\right.$

$2) x))+K_{t e}\left(-\frac{1}{2(j-1)} \cos ((j-1) x)+\frac{1}{2(j+1)} \cos ((j+1) x)\right)+K_{r e}\left(\frac{1}{2(j-1)} \sin ((j-1) x)+\frac{1}{2(j+1)} \sin ((j+1) x)\right]_{\phi_{\text {in }}}^{\phi_{\text {out }}}$

$b_{y 1}^{*}=-\frac{1}{\pi}\left[K_{t c} f_{z}\left(\frac{3}{4} \cos (x)-\frac{1}{12} \cos (3 x)\right)+K_{r c} f_{z}\left(\frac{1}{4} \sin (x)-\frac{1}{12} \sin (3 x)\right)+K_{t e}\left(-\frac{1}{2} x+\frac{1}{4} \sin (2 x)\right)+K_{r e}\left(-\frac{1}{4} \cos (2 x)\right]_{\phi_{\text {in }}}^{\phi_{\text {out }}}\right.$

$b_{y 2}^{*}=-\frac{1}{\pi}\left[K_{t c} f_{z}\left(\frac{1}{4} \cos (2 x)-\frac{1}{16} \cos (4 x)\right)+K_{r c} f_{z}\left(\frac{1}{4} x-\frac{1}{16} \sin (4 x)\right)+K_{t e}\left(-\frac{1}{2} \sin (x)+\frac{1}{6} \sin (3 x)\right)+K_{r e}\left(-\frac{1}{2} \cos (x)-\right.\right.$

$\left.\frac{1}{6} \cos (3 x)\right]_{\phi_{\text {in }}}^{\phi_{\text {out }}}$

$b_{y j}^{*}=-\frac{1}{\pi}\left[K_{t c} f_{z}\left(\frac{1}{2 j} \cos (j x)-\frac{1}{4(j-2)} \cos ((j-2) x)-\frac{1}{4(j+2)} \cos ((j+2) x)\right)+K_{r c} f_{z}\left(\frac{1}{4(j-2)} \sin ((j-2) x)-\frac{1}{4(j+2)} \sin ((j+\right.\right.$

$2) x))+K_{t e}\left(-\frac{1}{2(j-1)} \sin ((j-1) x)+\frac{1}{2(j+1)} \sin ((j+1) x)\right)+K_{r e}\left(-\frac{1}{2(j-1)} \cos ((j-1) x)-\frac{1}{2(j+1)} \cos ((j+1) x)\right]_{\phi_{\text {in }}}^{\phi_{\text {out }}}$

$\underline{\text { Coefficients for total cutting force without edge coefficients }}$

$a_{\text {tot } 0}^{* *}=-\frac{1}{2 \pi}[(\cos (x))]_{\phi_{\text {in }}}^{\phi_{\text {out }}}$

$a_{\text {tot } 1}^{* *}=-\frac{1}{\pi}\left[\left(\frac{1}{4} \cos (2 x)\right)\right]_{\phi_{\text {in }}}^{\phi_{\text {out }}}$

$a_{\text {totj }}^{* *}=-\frac{1}{\pi}\left[\left(-\frac{1}{2(j-1)} \cos ((j-1) x)+\frac{1}{2(j+1)} \cos ((j+1) x)\right)\right]_{\phi_{\text {in }}}^{\phi_{\text {out }}}$

$b_{\text {tot } 1}^{* *}=-\frac{1}{\pi}\left[\left(-\frac{x}{2}+\frac{1}{4} \sin (2 x)\right)\right]_{\phi_{\text {in }}}^{\phi_{\text {out }}}$

$b_{\text {totj }}^{* *}=-\frac{1}{\pi}\left[\left(-\frac{1}{2(j-1)} \sin ((j-1) x)+\frac{1}{2(j+1)} \sin ((j+1) x)\right)\right]_{\phi_{\text {in }}}^{\phi_{\text {int }}}$ 